\title{
Bounds for the Hückel energy of a graph
}

\author{
Ebrahim Ghorbani ${ }^{1,2, *}$, Jack H. Koolen ${ }^{3,4, \dagger}$, Jae Young Yang ${ }^{3, \ddagger}$ \\ e_ghorbani@math.sharif.edu koolen@postech.ac.kr rafle@postech.ac.kr \\ ${ }^{1}$ Department of Mathematical Sciences, Sharif University of Technology, \\ P.O. Box 11155-9415, Tehran, Iran \\ ${ }^{2}$ School of Mathematics, Institute for Research in Fundamental Sciences (IPM), \\ P.O. Box 19395-5746, Tehran, Iran \\ ${ }^{3}$ Department of Mathematics, POSTECH, Pohang 790-785, South Korea \\ ${ }^{4}$ Pohang Mathematics Institute, POSTECH, Pohang 790-785, South Korea \\ Submitted: Oct 9, 2009; Accepted: Oct 25, 2009; Published: Nov 7, 2009 \\ Mathematics Subject Classifications: 05C50, 05E30
}

\begin{abstract}
Let $G$ be a graph on $n$ vertices with $r:=\lfloor n / 2\rfloor$ and let $\lambda_{1} \geqslant \cdots \geqslant \lambda_{n}$ be adjacency eigenvalues of $G$. Then the Hückel energy of $G, \operatorname{HE}(G)$, is defined as

$$
\operatorname{HE}(G)= \begin{cases}2 \sum_{i=1}^{r} \lambda_{i}, & \text { if } n=2 r \\ 2 \sum_{i=1}^{r} \lambda_{i}+\lambda_{r+1}, & \text { if } n=2 r+1 .\end{cases}
$$

The concept of Hückel energy was introduced by Coulson as it gives a good approximation for the $\pi$-electron energy of molecular graphs. We obtain two upper bounds and a lower bound for $\operatorname{HE}(G)$. When $n$ is even, it is shown that equality holds in both upper bounds if and only if $G$ is a strongly regular graph with parameters $(n, k, \lambda, \mu)=\left(4 t^{2}+4 t+2,2 t^{2}+3 t+1, t^{2}+2 t, t^{2}+2 t+1\right)$, for positive integer $t$. Furthermore, we will give an infinite family of these strongly regular graph whose construction was communicated by Willem Haemers to us. He attributes the construction to J.J. Seidel.
\end{abstract}

*This work was done while the first author was visiting the department of mathematics of POSTECH. He would like to thank the department for its hospitality and support.

${ }^{\dagger} \mathrm{JHK}$ was partially supported by a grant from the Korea Research Foundation funded by the Korean government (MOEHRD) under grant number KRF-2007-412-J02302.

$\ddagger$ The results in this paper were obtained as a part of an Undergraduate Research Project of POSTECH under project number 2.0005656.01 and JYY greatly thanks POSTECH for its support. 


\section{Introduction}

Throughout this paper, all graphs are simple and undirected. Let $G$ be a graph with $n$ vertices and $A$ be the adjacency matrix of $G$. Then the eigenvalues of $G$ are defined as the eigenvalues of $A$. As all eigenvalues of $A$ are real, we can rearrange them as $\lambda_{1} \geqslant \cdots \geqslant \lambda_{n}$. I. Gutman (see [9]) defined the energy of $G, \mathrm{E}(G)$, by

$$
\mathrm{E}(G):=\sum_{i=1}^{n}\left|\lambda_{i}\right|
$$

In chemistry, the energy of a given molecular graph is of interest since it can be related to the total $\pi$-electron energy of the molecule represented by that graph. The reason for Gutman's definition is that $\mathrm{E}(G)$ gives a good approximation for the $\pi$-electron energy of a molecule where $G$ is then the corresponding molecular graph. For a survey on the energy of graphs, see [9]. The Hückel energy of $G$, denoted by $\operatorname{HE}(G)$, is defined as

$$
\mathrm{HE}(G)= \begin{cases}2 \sum_{i=1}^{r} \lambda_{i}, & \text { if } n=2 r \\ 2 \sum_{i=1}^{r} \lambda_{i}+\lambda_{r+1}, & \text { if } n=2 r+1 .\end{cases}
$$

The idea of introducing Hückel energy (implicitly) exists in Erich Hückel's first paper [10] in 1931 and is also found in his book [11]. The concept was explicitly used in 1940 by Charles Coulson [2] but, most probably, can be found also in his earlier articles. In a "canonical" form, the theory behind the Hückel energy was formulated in a series of papers by Coulson and Longuet-Higgins, of which the first (and most important) is [3]. In comparison with energy, the Hückel energy of a graph gives a better approximation for the total $\pi$-electron energy of the molecule represented by that graph, see [7]. Clearly for a graph $G$ vertices, $\operatorname{HE}(G) \leqslant \mathrm{E}(G)$, and if $G$ is bipartite, then equality holds. Koolen and Moulton in $[12,13]$ gave upper bounds on the energy of graphs and bipartite graphs, respectively. These bounds have been generalized in several ways. Obviously, the upper bounds of Koolen and Moulton also give upper bounds for the Hückel energy of graphs. In this paper, we obtain better upper bounds for the Hückel energy of a graph. More precisely, we prove that for a graph $G$ with $n$ vertices and $m$ edges,

$$
\mathrm{HE}(G) \leqslant \begin{cases}\frac{2 m}{n-1}+\frac{\sqrt{2 m(n-2)\left(n^{2}-n-2 m\right)}}{n-1}, & \text { if } m \leqslant \frac{n^{3}}{2(n+2)} \\ \frac{2}{n} \sqrt{m n\left(n^{2}-2 m\right)}<\frac{4 m}{n}, & \text { otherwise; }\end{cases}
$$

if $n$ is even, and

$$
\mathrm{HE}(G) \leqslant \begin{cases}\frac{2 m}{n-1}+\frac{\sqrt{2 m n\left(n^{2}-3 n+1\right)\left(n^{2}-n-2 m\right)}}{n(n-1)} & \text { if } m \leqslant \frac{n^{2}(n-3)^{2}}{2\left(n^{2}-4 n+11\right)} \\ \frac{1}{n} \sqrt{2 m(2 n-1)\left(n^{2}-2 m\right)}, & \text { otherwise; }\end{cases}
$$

if $n$ is odd. Then we show that

$$
\mathrm{HE}(G) \leqslant \frac{n}{2}(1+\sqrt{n-1})
$$


if $n$ is even, and

$$
\mathrm{HE}(G)<\frac{n}{2}\left(1+\sqrt{n}-\frac{1}{\sqrt{n}}\right),
$$

if $n$ is odd. Moreover, equality is attained in (1) if and only if equality attained in (3) if and only if $G$ is a strongly regular graph with parameters $(n, k, \lambda, \mu)=\left(4 t^{2}+4 t+2,2 t^{2}+\right.$ $\left.3 t+1, t^{2}+2 t, t^{2}+2 t+1\right)$. The proofs of the above upper bounds are given in Section 2 . It is known that $\mathrm{E}(G) \geqslant 2 \sqrt{n-1}$ for any graph $G$ on $n$ vertices with no isolated vertices with equality if and only if $G$ is the star $K_{1, n-1}$ (see [4]). In Section 3, we prove that the same bound holds for Hückel energy. In the last section, we give a construction of $\operatorname{srg}\left(4 t^{2}+4 t+2,2 t^{2}+3 t+1, t^{2}+2 t, t^{2}+2 t+1\right)$.

\section{The upper bound for Hückel energy}

In this section we prove (1), (2), (3), and (4). The equality cases are also discussed. We begin by stating a lemma which will be used later.

Lemma 1. Let $G$ be a graph with $n$ vertices and $m$ edges. Suppose $r:=\lfloor n / 2\rfloor$ and

$$
\alpha:=\sum_{i=1}^{r} \lambda_{i}^{2}(G) .
$$

If $m \geqslant n-1 \geqslant 2$, then

$$
\frac{\alpha}{r} \leqslant \frac{4 m^{2}}{n^{2}}
$$

Proof. First, suppose $m \geqslant n$. Then $G$ contains a cycle, and so by interlacing, we see $\lambda_{n}^{2}+\lambda_{n-1}^{2} \geqslant 2$. Therefore, $\alpha / r \leqslant(2 m-2) / r \leqslant 4 m^{2} / n^{2}$. If $m=n-1$ and $G$ is connected, then $G$ is a tree. Thus $\alpha=m$, and obviously (5) holds. So in the rest of proof we assume that $G$ is disconnected and $m=n-1$. If $G$ has at least three non-trivial components, then at least one of the components contains a cycle. The component containing a cycle has either an eigenvalue at most $\leqslant-2$, or two eigenvalues $\leqslant-1$ and the other two components have an eigenvalue $\leqslant-1$. It turns out that $\lambda_{n}^{2}+\lambda_{n-1}^{2}+\lambda_{n-2}^{2}+\lambda_{n-3}^{2} \geqslant 4$ where $n \geqslant 7$. Hence

$$
\alpha \leqslant 2 m-4 .
$$

It is easily seen that (5) follows from (6). Now, suppose that $G$ has two non-trivial connected components $G_{1}$ and $G_{2}$, say. Let $G_{1}, G_{2}$ have $n_{1}, n_{2}$ vertices and $m_{1}, m_{2}$ edges, respectively. First suppose $n_{1}, n_{2} \geqslant 3$. If $G_{1}$ or $G_{2}$ contains a $K_{1,2}$ as an induced subgraph, we are done by interlacing. So one may assume that both $G_{1}$ and $G_{2}$ contain a triangle. It turns out that $m_{1} \geqslant n_{1}$ and $m_{2} \geqslant n_{2}$. Hence $G$ must have an isolated vertex which implies $n \geqslant 7$. On the other hand, by interlacing, the four smallest eigenvalues of $G$ are at most -1 implying (6). Now, assume that $n_{1}=2$. So $G_{2}$ must contain a cycle $C_{\ell}$. We may assume that $C_{\ell}$ has no chord. If $\ell \geqslant 4$, we are done. So let $\ell=3$. If $n_{2}=3$, then $G$ does not have isolated vertices, i.e., $G=C_{3} \cup K_{2}$, for which (5) holds. Thus $n_{2} \geqslant 4$ 

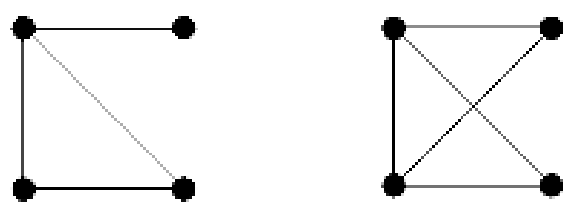

Figure 1: The paw and the diamond graphs

which means that at least one of the diamond graph, the paw graph (see Figure 1), or $K_{4}$ is induced subgraph of $G$. If it contains either the diamond or the paw graph, we are done by interlacing. If it contains $K_{4}$, then $G$ must have at least two isolated vertices, i.e., $n \geqslant 8$. Thus the four smallest eigenvalues of $G$ are at most -1 which implies (6). Finally, assume that $G$ has exactly one non-trivial component $G_{1}$ with $n_{1}$ vertices. It turns out that $n_{1} \geqslant 3$ and $G_{1}$ contains a cycle. By looking at the table of graph spectra of [5, pp. 272-3], it is seen that if $n_{1}=3,4, G$ satisfies (5). If $n_{1} \geqslant 5$, then making use of the table mentioned above and interlacing it follows that $\lambda_{n}^{2}+\lambda_{n-1}^{2}+\lambda_{n-2}^{2} \geqslant 4$ unless $G_{1}$ is a complete graph in which case $G$ has at least 11 vertices and the four smallest eigenvalues of $G$ is -1 , implying the result.

\subsection{Even order graphs}

In this subsection we prove (1) and (3) for graphs of an even order. The cases of equalities are also characterized.

Theorem 2. Let $G$ be a graph on $n$ vertices and $m$ edges where $n$ is even. Then (1) holds. Moreover, equality holds if and only if $n=4 t^{2}+4 t+2$ for some positive integer $t, m=\left(2 t^{2}+2 t+1\right)\left(2 t^{2}+3 t+1\right)$ and $G$ is a strongly regular graph with parameters $(n, k, \lambda, \mu)=\left(4 t^{2}+4 t+2,2 t^{2}+3 t+1, t^{2}+2 t, t^{2}+2 t+1\right)$.

Proof. Let $n=2 r$ and $\lambda_{1} \geqslant \lambda_{2} \geqslant \cdots \geqslant \lambda_{n}$ be the eigenvalues of $G$. Then

$$
\sum_{i=1}^{n} \lambda_{i}=0 \quad \text { and } \quad \sum_{i=1}^{n} \lambda_{i}^{2}=2 m .
$$

Let $\alpha$ be as in Lemma 1 . Then

$$
2 m-\alpha=\sum_{i=r+1}^{n} \lambda_{i}^{2}
$$

and $\lambda_{1}^{2} \leqslant \alpha \leqslant 2 m-2$. By the Cauchy-Schwartz inequality,

$$
\mathrm{HE}(G)=2 \sum_{i=1}^{r} \lambda_{i} \leqslant 2 \lambda_{1}+2 \sqrt{(r-1)\left(\alpha-\lambda_{1}^{2}\right)} .
$$


The function $x \mapsto x+\sqrt{(r-1)\left(\alpha-x^{2}\right)}$ decreases on the interval $\sqrt{\alpha / r} \leqslant x \leqslant \sqrt{\alpha}$. By Lemma $1, m / r \geqslant \sqrt{\alpha / r}$. Since $\lambda_{1} \geqslant m / r$, we see that

$$
\operatorname{HE}(G) \leqslant f_{1}(\alpha):=\frac{2 m}{r}+2 \sqrt{(r-1)\left(\alpha-m^{2} / r^{2}\right)} .
$$

On the other hand,

$$
\operatorname{HE}(G)=-2 \sum_{i=r+1}^{n} \lambda_{i} \leqslant f_{2}(\alpha):=2 \sqrt{r(2 m-\alpha)} .
$$

Let

$$
f(\alpha):=\min \left\{f_{1}(\alpha), f_{2}(\alpha)\right\} .
$$

We determine the maximum of $f$. We observe that $f_{1}$ and $f_{2}$ are increasing and decreasing functions in $\alpha$, respectively. Therefore, $\max f=f\left(\alpha_{0}\right)$ where $\alpha_{0}$ is the unique point with $f_{1}\left(\alpha_{0}\right)=f_{2}\left(\alpha_{0}\right)$. So we find the solution of the equation $f_{1}(\alpha)=f_{2}(\alpha)$. To do so, let $\sigma:=\sqrt{\alpha-m^{2} / r^{2}}$ and consider the equation

$$
\frac{m}{r}+\sigma \sqrt{r-1}=\sqrt{r\left(2 m-\sigma^{2}-m^{2} / r^{2}\right)} .
$$

This equation has the roots

$$
\sigma_{1,2}:=\frac{-m \sqrt{r-1} \pm r \sqrt{2 m\left(2 r^{2}-r-m\right)}}{r(2 r-1)} .
$$

If $m \leqslant 2 r^{3} /(r+1)$, then $\sigma_{1} \geqslant 0$ and so

$$
\begin{aligned}
\mathrm{HE}(G) & \leqslant \frac{2 m}{r}+\frac{2 \sqrt{r-1}}{r(2 r-1)}\left(-m \sqrt{r-1}+r \sqrt{2 m\left(2 r^{2}-r-m\right)}\right) \\
& =\frac{2 m}{n-1}+\frac{\sqrt{2 m(n-2)\left(n^{2}-n-2 m\right)}}{n-1}
\end{aligned}
$$

otherwise

$$
\operatorname{HE}(G) \leqslant 2 \sqrt{r\left(2 m-m^{2} / r^{2}\right)},
$$

which is less than $4 m / n$ for $m>2 r^{3} /(r+1)$. This shows that Inequality (1) holds.

Now let us consider the case that equality is attained in (1). First let $m \leqslant \frac{2 r^{3}}{r+1}$. Then equality holds if and only if

1. $\lambda_{1}=\frac{m}{r}$;

2. $\lambda_{2}=\cdots=\lambda_{r}=\frac{\sigma_{1}}{\sqrt{r-1}}$

3. $\lambda_{r+1}=\cdots=\lambda_{n}=-\frac{1}{\sqrt{r}} \sqrt{2 m-\sigma_{1}^{2}-m^{2} / r^{2}}$. 
The first condition shows that $G$ must be $\frac{m}{r}$-regular, and the second and third conditions imply that $G$ must be strongly regular graph as a regular graph with at most three distinct eigenvalues is strongly regular, cf. [8, Lemma 10.2.1]. From [8, Lemma 10.3.5] it follows that $G$ has to have the parameters as required in the theorem. If $m>\frac{2 r^{3}}{r+1}$, then with the same reasoning as above one can show that $G$ has to be strongly regular graph with eigenvalue 0 of multiplicity $r-1$ and by [8, Lemma 10.3.5] such a graph does not exist.

Optimizing the Hückel energy over the number of edges we obtain:

Theorem 3. Let $G$ be a graph on $n$ vertices where $n$ is even. Then (3) holds. Equality holds if and only if $G$ is a strongly regular graph with parameters $\left(4 t^{2}+4 t+2,2 t^{2}+3 t+\right.$ $\left.1, t^{2}+2 t, t^{2}+2 t+1\right)$, for some positive integer $t$.

Proof. Suppose that $G$ is a graph with $n$ vertices and $m$ edges. If $m \leqslant n-2$, then (3) obviously holds as $\mathrm{E}(G) \leqslant 2 m$ (see [9]). If $m \geqslant n-1$, then using routine calculus, it is seen that the right hand side of (9) - considered as a function of $m$-is maximized when

$$
m=n(n-1+\sqrt{n-1}) / 4 .
$$

Inequality (3) now follows by substituting this value of $m$ into (1). (We note that the maximum of the right hand side of (10) is $2 n^{3} /(n+2)$ which is less than the above maximum.) Moreover, from Theorem 2 it follows that equality holds in (3) if and only if $G$ is a strongly regular graph with parameters $\left(4 t^{2}+4 t+2,2 t^{2}+3 t+1, t^{2}+2 t, t^{2}+2 t+1\right)$.

\subsection{Odd order graphs}

In this subsection we prove (1) and (4) for graphs of an odd order and discuss the equality case and tightness of the bounds.

Theorem 4. Let $m \geqslant n-1 \geqslant 3$ and $G$ be a graph with $n$ vertices and $m$ edges where $n$ is odd. Then (2) holds.

Proof. Let $n=2 r+1, \alpha$ be as before, and

$$
\beta:=\lambda_{r+1} .
$$

We have

$$
2 m-\alpha \geqslant(r+1) \beta^{2} .
$$

This obviously holds if $\beta \leqslant 0$. For $\beta \geqslant 0$ it follows from the following:

$$
\begin{aligned}
2 m-\alpha-\beta^{2} & =\sum_{i=r+2}^{n} \lambda_{i}^{2} \geqslant \frac{1}{r}\left(\sum_{i=r+2}^{n} \lambda_{i}\right)^{2} \\
& =\frac{1}{r}\left(\sum_{i=1}^{r+1} \lambda_{i}\right)^{2} \geqslant \frac{1}{r}(r+1)^{2} \beta^{2},
\end{aligned}
$$

THE ELECTRONiC JouRnal of COMBinatorics 16 (2009), \#R134 
where the first inequality follows from the Cauchy-Schwartz inequality. In a similar manner as the proof of Theorem 2 , we find that $\operatorname{HE}(G) \leqslant \min \left\{f_{1}(\alpha, \beta), f_{2}(\alpha, \beta)\right\}$, where

$$
\begin{aligned}
& f_{1}(\alpha, \beta)=4 m / n+2 \sqrt{(r-1)\left(\alpha-4 m^{2} / n^{2}\right)}+\beta, \text { and } \\
& f_{2}(\alpha, \beta)=2 \sqrt{r\left(2 m-\alpha-\beta^{2}\right)}-\beta .
\end{aligned}
$$

Let

$$
f(\alpha, \beta):=\min \left\{f_{1}(\alpha, \beta), f_{2}(\alpha, \beta)\right\} .
$$

We determine the maximum of $f$ over the compact set

$$
D:=\left\{(\alpha, \beta): \alpha \geqslant 4 m^{2} / n^{2}, 2 m-(r+1) \beta^{2} \geqslant \alpha\right\} .
$$

Note that for $(\alpha, \beta) \in D$ one has $-\beta_{0} \leqslant \beta \leqslant \beta_{0}$, where

$$
\beta_{0}=\frac{2}{n} \sqrt{\frac{m\left(n^{2}-2 m\right)}{n+1}} .
$$

Neither the gradient of $f_{1}$ nor that of $f_{2}$ has a zero in interior of $D$. So the maximum of $f$ occurs in the set

$$
L:=\left\{(\alpha, \beta): f_{1}(\alpha, \beta)=f_{2}(\alpha, \beta)\right\},
$$

where the gradient of $f$ does not exist or it occurs in the boundary of $D$ consisting of

$$
\begin{aligned}
& D_{1}:=\left\{(\alpha, \beta): \alpha=4 m^{2} / n^{2},-\beta_{0} \leqslant \beta \leqslant \beta_{0}\right\}, \\
& D_{2}:=\left\{(\alpha, \beta): \alpha=2 m-(r+1) \beta^{2},-\beta_{0} \leqslant \beta \leqslant \beta_{0}\right\} .
\end{aligned}
$$

For any $(\alpha, \beta) \in D, f_{2}(\alpha, \beta) \leqslant f_{2}\left(4 m^{2} / n^{2}, \beta\right)$. It is easily seen that the maximum of $f_{2}\left(4 m^{2} / n^{2}, \beta\right)$ occurs in

$$
\beta_{1}:=-\frac{1}{n} \sqrt{\frac{2 m\left(n^{2}-2 m\right)}{2 n-1}} .
$$

Therefore,

$$
\max f_{2}=f_{2}\left(4 m^{2} / n^{2}, \beta_{1}\right)=\frac{1}{n} \sqrt{2 m(2 n-1)\left(n^{2}-2 m\right)} .
$$

In the rest of proof, we determine $\max f$ for

$$
m \leqslant \frac{n^{2}(n-3)^{2}}{2\left(n^{2}-4 n+11\right)}
$$

if $m>\frac{n^{2}(n-3)^{2}}{2\left(n^{2}-4 n+11\right)}$, then (2) follows from (13).

On $D_{1}$, we have

$$
\max f_{\left.\right|_{D_{1}}} \leqslant f_{1}\left(4 m^{2} / n^{2}, \beta_{0}\right)=4 m / n+\beta_{0} .
$$

On $D_{2}$, one has

$$
\begin{aligned}
& f_{1}(\beta)=4 m / n+2 \sqrt{(r-1)\left(2 m-(r+1) \beta^{2}-4 m^{2} / n^{2}\right)}+\beta, \text { and } \\
& f_{2}(\beta)=(n-1)|\beta|-\beta .
\end{aligned}
$$


In order to find max $f_{\left.\right|_{D_{2}}}$, we look for the points where $f_{1}(\beta)=f_{2}(\beta)$. The solution of this equation for $\beta \leqslant 0$ is

$$
\beta_{2}=\frac{-2 m(n+1)-\sqrt{2 m n\left(n^{2}-2 n-3\right)\left(n^{2}-n-2 m\right)}}{n\left(n^{2}-1\right)},
$$

and for $\beta \geqslant 0$ is

$$
\beta_{3}=\frac{2 m(n-3)+\sqrt{2 m(n-3)\left(n^{4}-4 n^{3}-2 m n^{2}+3 n^{2}+6 m n-8 m\right)}}{n\left(n^{2}-4 n+3\right)} .
$$

We have $\beta_{2} \geqslant-\beta_{0}$ if and only if $m \leqslant \frac{n^{2}(n+1)}{2(n+3)}$, and $\beta_{3} \leqslant \beta_{0}$ if and only if $m \leqslant \frac{n^{2}(n-3)^{2}}{2\left(n^{2}-4 n+11\right)}$. Moreover $f_{2}\left(\beta_{2}\right)>f_{2}\left(\beta_{3}\right)$. Thus if $m \leqslant \frac{n^{2}(n-3)^{2}}{2\left(n^{2}-4 n+11\right)}$,

$$
\max f_{\left.\right|_{D_{2}}}=f_{2}\left(\beta_{2}\right)=\frac{2 m(n+1)+\sqrt{2 m n\left(n^{2}-2 n-3\right)\left(n^{2}-n-2 m\right)}}{n^{2}-1} .
$$

Now we examine $\max f_{\left.\right|_{L}}$. Let $\sigma:=\sqrt{\alpha-4 m^{2} / n^{2}}$. To determine $(\alpha, \beta)$ satisfying $f_{1}(\alpha, \beta)=f_{2}(\alpha, \beta)$ it is enough to find the zeros of the following quadratic form:

$$
(2 n-4) \sigma^{2}+4(2 m / n+\beta) \sqrt{n-3} \sigma+(4 m / n+2 \beta)^{2}-(n-1)\left(4 m-2 \beta^{2}-8 m^{2} / n^{2}\right) .
$$

The zeros are

$$
\begin{aligned}
\sigma_{1,2}:=\frac{1}{n(n-2)}[- & (2 m+n \beta) \sqrt{n-3} \pm \\
& \left.\sqrt{(n-1)\left(2 m n^{3}-\beta^{2} n^{3}+\beta^{2} n^{2}-4 m n^{2}-4 m \beta n-4 n m^{2}+4 m^{2}\right)}\right] .
\end{aligned}
$$

Note that $\sigma_{2}<0$ and so is not feasible. Let us denote the constant term of (15) by $h(\beta)$ as a function of $-\beta_{0} \leqslant \beta \leqslant \beta_{0}$. Then $\sigma_{1} \geqslant 0$ if and only if $h(\beta) \leqslant 0$. Moreover $h(\beta) \leqslant h\left(\beta_{0}\right)$, and $h\left(\beta_{0}\right) \leqslant 0$ if

$$
m \leqslant \frac{n^{2}(n-3)^{2}}{2\left(n^{2}-4 n+11\right)} .
$$

Thus, with this condition on $m, f_{1}$ becomes $f_{1}(\beta)=4 m / n+2 \sqrt{r-1} \sigma_{1}$. The roots of $f_{1}^{\prime}(\beta)=0$ are

$$
\beta_{4,5}=\frac{-2 m\left(n^{2}-3 n+1\right) \pm \sqrt{2 m n\left(n^{2}-3 n+1\right)\left(n^{2}-n-2 m\right)}}{n\left(n^{3}-4 n^{2}+4 n-1\right)} .
$$

It is seen that $-\beta_{0} \leqslant \beta_{5} \leqslant \beta_{4} \leqslant 0$ unless $m \leqslant n^{2} /(2 n-2)$. We have

$$
f_{1}\left(\beta_{4}\right)-f_{1}\left(\beta_{5}\right)=\frac{2 \sqrt{2 m n\left(n^{2}-3 n+1\right)\left(n^{2}-n-2 m\right)}}{n(n-2)\left(n^{3}-4 n^{2}+4 n-1\right)} .
$$


Thus $f_{1}\left(\beta_{4}\right) \geqslant f_{1}\left(\beta_{5}\right)$. It turns out that $f_{1}$ decreases for $\beta \geqslant \beta_{4}$, so $f_{1}\left(\beta_{4}\right) \geqslant f_{1}\left(\beta_{0}\right)$. It is easily seen that $f_{1}\left(\beta_{4}\right) \geqslant f_{1}\left(-\beta_{0}\right)$. Therefore, for $m \leqslant \frac{n^{2}(n-3)^{2}}{2\left(n^{2}-4 n+11\right)}$ we have

$$
\max f_{\left.\right|_{L}}=f_{1}\left(\beta_{4}\right)=\frac{2 m}{n-1}+\frac{\sqrt{2 m n\left(n^{2}-3 n+1\right)\left(n^{2}-n-2 m\right)}}{n(n-1)} .
$$

The result follows from comparing the three maxima max $f_{\left.\right|_{1}}$, max $f_{\left.\right|_{D_{2}}}$, and $\max f_{\left.\right|_{L}}$.

Theorem 5. Let $G$ be a graph on $n$ vertices where $n$ is odd. Then (4) holds.

Proof. The maximum of the right hand side of (13) - as a function of $m$ - is

$$
\frac{n(n-3) \sqrt{2(n+1)(2 n-1)}}{n^{2}-4 n+11},
$$

which is obtained when $m$ is given by (14). Also, the right hand side of (16) is maximized when

$$
m=\frac{n(n-1+\sqrt{n})}{4} .
$$

This maximum value is equal to $\frac{n}{2}\left(1+\sqrt{n}-\frac{1}{\sqrt{n}}\right)$ which is greater than (17). This completes the proof.

Remark 6. Here we show that no graph can attain the bound in (2). Let us keep the notation of the proof of Theorem 4. First we consider $m>\frac{1}{2} n^{2}(n-3)^{2} /\left(n^{2}-4 n+11\right)$. Therefore, $\operatorname{HE}(G)$ equals (13). Then $\alpha=4 m^{2} / n^{2}$ and $\lambda_{r+1}=\beta_{1}$. This means that $G$ is a regular graph with only one positive eigenvalue. Then by [5, Theorem 6.7], $G$ is a complete multipartite graph. As the rank of a complete multipartite graph equals the number of its parts, $G$ must have $r+2$ parts. Such a graph cannot be regular, a contradiction. Now, we consider $m \leqslant \frac{1}{2} n^{2}(n-3)^{2} /\left(n^{2}-4 n+11\right)$. Hence $\operatorname{HE}(G)$ is equal to (16). Then $G$ must be a regular graph of degree $k$, say, with $\lambda_{2}=\cdots=\lambda_{r}, \lambda_{r+1}=\beta_{4}$, and $\lambda_{r+2}=\cdots=\lambda_{n}$. Since $\lambda_{r+1}=\beta_{4}<0, \lambda_{2}$ and $\lambda_{n}$ have different multiplicities, and thus all eigenvalues of $G$ are integral. Let $\lambda_{2}=t$. Then $\lambda_{n}=-t-s$, for some integer $s \geqslant 0$. It follows that $k+\lambda_{r+1}=t+r s$. This implies that either $s=0$ or $s=1$. If $s=0$, then $k+\lambda_{r+1}=t$, and so $\operatorname{HE}(G)=k+(n-2) t$. This must be equal to (16) which implies

$$
t=\frac{k+\sqrt{n k\left(n^{2}-3 n+1\right)(n-1-k)}}{n^{2}-3 n+2} .
$$

Substituting this value of $t$ in the equation $n k=k^{2}+(n-2) t^{2}+(t-k)^{2}$ and solving in terms of $k$ yields $k=n /(n-1)$ which is impossible. If $s=1$, then $k+\lambda_{r+1}=t+r$, and so $\operatorname{HE}(G)=k+(n-2) t+r$. It follows that

$$
t=\frac{k-(n-1)^{2} / 2+\sqrt{n k\left(n^{2}-3 n+1\right)(n-1-k)}}{n^{2}-3 n+2} .
$$

Substituting this value of $t$ in the equation $n k=k^{2}+(r-1) t^{2}+r(t+1)^{2}+(r+t-k)^{2}$ and solving in terms of $k$ yields $k=(n-1+\sqrt{n}) / 2$ which implies $t=(\sqrt{n}-1) / 2$. Therefore, we have $\lambda_{r+1}=-\frac{1}{2}$, a contradiction. 
Remark 7. Note that a conference strongly regular graph $G$, i.e, a $\operatorname{srg}(4 t+1,2 t, t-1, t)$, has spectrum $\left\{[2 t]^{1},[(-1+\sqrt{4 t+1}) / 2]^{2 t},[(-1-\sqrt{4 t+1}) / 2]^{2 t}\right\}$, and hence $\operatorname{HE}(G)=$ $\frac{2 t+1}{2}(1+\sqrt{4 t+1})$. This is about half of the upper bound given in (4). For odd order graphs, we can come much closer to (4). Let $G$ be a $\operatorname{srg}\left(4 t^{2}+4 t+2,2 t^{2}+3 t+1, t^{2}+2 t, t^{2}+2 t+1\right)$. If one adds a new vertex to $G$ and join it to neighbors of some fixed vertex of $G$, then the resulting graph $H$ has the spectrum

$$
\left\{\left[\lambda_{1}\right]^{1},[t]^{2 t^{2}+2 t-1},\left[\lambda_{2}\right]^{1},[0]^{1},[-t-1]^{2 t^{2}+2 t},\left[\lambda_{3}\right]^{1}\right\}
$$

where $\lambda_{1}, \lambda_{2}, \lambda_{3}$ are the roots of the polynomial

$$
p(x):=x^{3}-\left(2 t^{2}+3 t\right) x^{2}-\left(5 t^{2}+7 t+2\right) x+4 t^{4}+10 t^{3}+8 t^{2}+2 t .
$$

It turns out that $p(-\sqrt{2} t)=(4+3 \sqrt{2}) t^{3}+(8+7 \sqrt{2}) t^{2}+(2+2 \sqrt{2}) t$. Hence $\lambda_{3}<-\sqrt{2} t$ and so

$$
\mathrm{HE}(H)>2\left(2 t^{2}+2 t\right)(t+1)+2 \sqrt{2} t=\frac{4 t^{2}+4 t+3}{2}\left(\sqrt{4 t^{2}+4 t+3}\right)+O\left(4 t^{2}+4 t+3\right) .
$$

This shows that (4) is asymptotically tight.

\section{Lower bound}

It is known that $\mathrm{E}(G) \geqslant 2 \sqrt{n-1}$ for any graph $G$ on $n$ vertices with no isolated vertices with equality if and only if $G$ is the star $K_{1, n-1}$ (see [4]). Below we show that this is also the case for Hückel energy.

Theorem 8. For any graph $G$ on $n$ vertices with no isolated vertices,

$$
\mathrm{HE}(G) \geqslant 2 \sqrt{n-1}
$$

Equality holds if and only if $G$ is the star $K_{1, n-1}$.

Proof. If $G_{1}, G_{2}$ are two graphs with $n_{1}, n_{2}$ vertices, then $\operatorname{HE}\left(G_{1} \cup G_{2}\right) \geqslant \operatorname{HE}\left(G_{1}\right)+$ $\operatorname{HE}\left(G_{2}\right)$, and $\sqrt{n_{1}-1}+\sqrt{n_{1}-1} \geqslant \sqrt{n_{1}+n_{2}-1}$ for $n_{1}, n_{2} \geqslant 2$. This alows us to assume that $G$ is connected. The theorem clearly holds for $n \leqslant 3$, so suppose $n \geqslant 4$. Let $p, q$ be the number of positive and negative eigenvalues of $G$, respectively. Let $n$ be even; the theorem follows similarly for odd $n$. If $p=1$, then by [5, Theorem 6.7], $G$ is a complete multipartite graphs with $s$ parts, say. If $s \leqslant \frac{n}{2}+1, \operatorname{HE}(G)=E(G)$ and we are done, so let $s \geqslant \frac{n}{2}+2$. Note that the complete graph $K_{s}$ is an induced subgraph of $G$, so by interlacing, $\lambda_{n-s+2} \leqslant-1$. Therefore $\lambda_{\frac{n}{2}} \leqslant-1$ and thus $\operatorname{HE}(G) \geqslant n$ and this is greater than $2 \sqrt{n-1}$ for $n \geqslant 3$. So we may assume that $p \geqslant 2$. This implies that $q \geqslant 2$ as well. 
Note that either $p \leqslant \frac{n}{2}+2$ or $q \leqslant \frac{n}{2}+2$. Suppose $q \leqslant \frac{n}{2}+2$; the proof is similar for the other case. If we also have $p \leqslant \frac{n}{2}+2$, we are done. So let $p \geqslant \frac{n}{2}+2$. We observe that

$$
\begin{aligned}
\mathrm{E}(G) & =2\left(\lambda_{1}+\cdots+\lambda_{p}\right) \\
& \leqslant 2\left(\lambda_{1}+\cdots+\lambda_{r}\right)+2\left(\lambda_{p-2 r}+\cdots+\lambda_{r}\right) \\
& \leqslant \operatorname{HE}(G)+\frac{p-r}{r} \operatorname{HE}(G) .
\end{aligned}
$$

It turns out that

$$
\mathrm{HE}(G) \geqslant \frac{n}{2 p} \mathrm{E}(G) .
$$

On the other hand, we see that $\mathrm{E}(G) \geqslant p+2$, as the energy of any graph is at least the rank of its adjacency matrix ([6], see also [1]). Combining the above inequalities we find

$$
\begin{aligned}
\mathrm{HE}(G) & \geqslant \frac{n}{2 p}(p+2) \\
& =\frac{n}{2}+\frac{n}{p} \\
& \geqslant \frac{n^{2}}{2(n-2)},
\end{aligned}
$$

and the last value is greater than $2 \sqrt{n-1}$ for $n \geqslant 4$.

\section{A construction of $\operatorname{srg}\left(4 t^{2}+4 t+2,2 t^{2}+3 t+1, t^{2}+2 t\right.$, $\left.t^{2}+2 t+1\right)$}

In this section we give an infinite family of strongly regular graphs with parameters $(n, k, \lambda, \mu)=\left(4 t^{2}+4 t+2,2 t^{2}+3 t+1, t^{2}+2 t, t^{2}+2 t+1\right)$ whose construction was communicated by Willem Haemers to us. He attributes the construction to J. J. Seidel.

Let $G$ be a graph with vertex set $X$, and $Y \subseteq X$. From $G$ we obtain a new graph by leaving adjacency and non-adjacency inside $Y$ and $X \backslash Y$ as it was, and interchanging adjacency and non-adjacency between $Y$ and $X \backslash Y$. This new graph is said to be obtained by Seidel switching with respect to the set of vertices $Y$.

Let $q=2 t+1$ be a prime power. Let $\Gamma$ be the Paley graph of order $q^{2}$, that is, the graph with vertex set $\operatorname{GF}\left(q^{2}\right)$ and $x \sim y$ if $x-y$ is not a square in $\operatorname{GF}\left(q^{2}\right)$. Let $x$ be a primitive element of $\mathrm{GF}\left(q^{2}\right)$ and consider $V=\left\{x^{i(q+1)} \mid i=0, \ldots, q-1\right\} \cup\{0\}$. Then $V$ is the subfield $\operatorname{GF}(q)$ of $\operatorname{GF}\left(q^{2}\right)$ and $V$ forms a coclique of size $q$. Now $\left\{a+V \mid a \in \operatorname{GF}\left(q^{2}\right)\right\}$ forms a partition into $q$ cocliques of size $q$. Add an isolated vertex and apply the Seidel switching with respect to the union of $t$ disjoint cocliques. The resulting graph is a strongly regular graph with parameters $\left(4 t^{2}+4 t+2,2 t^{2}+t, t^{2}-1, t^{2}\right)$. Taking the complement of this graph give us a strongly regular graph with the required parameters. Note that for $t=1$, there exists only one such a graph namely the Johnson graph $J(5,2)$, for $t=2$ there are 10 . For $t \leqslant 9$ they do exist and $t=10$ seems the smallest open case. 
Acknowledgements. We are very grateful for the discussions with Patrick Fowler and Ivan Gutman. Partick Fowler suggested the name of Hückel energy and provided us with the reference [7]. Ivan Gutman presented us the history of the Hückel energy and provided us with the references $[2,3,10,11]$.

\section{References}

[1] S. Akbari, E. Ghorbani, and S. Zare, Some relations between rank, chromatic number and energy of graphs, Discrete Math. 309 (2009), 601-605

[2] C.A. Coulson, On the calculation of the energy in unsaturated hydrocarbon molecules, Proc. Cambridge Phil. Soc. 36 (1940), 201-203.

[3] C.A. Coulson and H.C. Longuet-Higgins, The electronic structure of conjugated systems. I. General theory, Proc. Roy. Soc. London A 191 (1947), 39-60.

[4] G. Caporossi, D. Cvetković, I. Gutman, and P. Hansen, Variable neighborhood search for extremal graphs. 2. Finding graphs with extremal energy, J. Chem. Inf. Comput. Sci. 39 (1999), 984-996.

[5] D.M. Cvetković, M. Doob, and H. Sachs, Spectra of Graphs, Theory and Applications, Third ed., Johann Ambrosius Barth, Heidelberg, 1995.

[6] S. Fajtlowicz, On conjectures of Graffiti. II, Eighteenth Southeastern International Conference on Combinatorics, Graph Theory, and Computing (Boca Raton, Fla., 1987). Congr. Numer. 60 (1987), 189-197.

[7] P.W. Fowler, Energies of graphs and molecules, in: Computational Methods in Modern Science and Enineering, Vol 2, parts A and B, Corfu, Greece, 2007, 517-520.

[8] C. Godsil and G. Royle, Algebraic Graph Theory, GTM 207, Springer, New York, 2001.

[9] I. Gutman, The energy of a graph: old and new results, in: Algebraic Combinatorics and Applications, A. Betten, A. Kohner, R. Laue, and A. Wassermann, eds., Springer, Berlin, 2001, 196-211.

[10] E. Hückel, Quantentheoretische Beiträge zum Benzolproblem, Z. Phys. 70 (1931), $204-286$.

[11] E. Hückel, Grundzüge der Theorie Ungesättigter und Aromatischer Verbindungen, Verlag Chemie, Berlin, 1940.

[12] J. Koolen and V. Moulton, Maximal energy graphs, Adv. in Appl. Math. 26 (2001), 47-51.

[13] J. Koolen and V. Moulton, Maximal energy bipartite graphs, Graphs Combin. 19 (2003), 131-135. 